\title{
A high-order perturbation of surfaces (HOPS) approach to Fokas integral equations: Vector electromagnetic scattering by periodic crossed gratings
}

\author{
David P. Nicholls ${ }^{\mathrm{a}, *}$, Venu Tammali ${ }^{\mathrm{a}}$ \\ ${ }^{a}$ Department of Mathematics, Statistics, and Computer Science, University of Illinois at Chicago, Chicago, IL \\ 60607
}

\begin{abstract}
The accurate simulation of linear electromagnetic scattering by diffraction gratings is crucial in many technologies of scientific and engineering interest. In this contribution we describe a HighOrder Perturbation of Surfaces (HOPS) algorithm built upon a class of Integral Equations due to the analysis of Fokas and collaborators, now widely known as the Unified Transform Method. The unknowns in this formalism are boundary quantities (the electric field and current at the layer interface) which are an order of magnitude fewer than standard volumetric approaches such as Finite Differences and Finite Elements. With detailed numerical experiments we show the efficiency, fidelity, and high-order accuracy one can achieve with an implementation of this algorithm.
\end{abstract}

Keywords: Layered media; Maxwell equations; Electromagnetic scattering; Integral equations; High-order spectral methods

\section{Introduction}

The scattering of linear electromagnetic waves by periodic diffraction gratings plays an important role in many applications. The robust and rapid capability of simulating these configurations numerically is of crucial importance, and in this contribution we focus upon the specific problem of vector electromagnetic radiation interacting with a periodic crossed diffraction grating (see Figure 1). A crossed grating is usually defined as two line gratings at right angles to each other, and we model this with a biperiodic layer interface (which permits non-orthogonal line intersection).

This model arises in a wide range of applications in optics and photonics, and of particular interest to this paper, in nanoplasmonics $[52,34,19]$. In this field one investigates topics as diverse as extraordinary optical transmission [18], surface enhanced spectroscopy [42], and surface plasmon resonance biosensing $[26,28,33,30,55,50]$.

Classical numerical algorithms such as Finite Elements (e.g., [59, 14, 27, 62]) and Finite Differences (e.g., $[11,58,32])$ have been used to simulate these configurations. However, these volumetric approaches are clearly disadvantaged with an unnecessarily large number of unknowns for the piecewise homogeneous grating problem we consider here. In addition, for these methods the unbounded problem domain must be truncated at some finite distance from the grating structure inducing the complication and error introduced by enforcing an (approximately) "Non-Reflecting Boundary Condition" (e.g., the Perfectly Matched Layer of Berenger [6] and variants of this, e.g., [21, 22, 23]).

Consequently, methods based upon Integral Equations (IEs) [13] are a natural candidate but face several challenges. For instance, specially designed quadrature rules must be designed to deliver high-order (spectral) accuracy, and these rules applied to these nonlocal IEs generate dense, non-symmetric positive definite linear systems to be solved. However, these issues have been satisfactorally addressed (possibly with the use of iterative solution procedures accelerated by Fast

\footnotetext{
* Corresponding author

Email addresses: davidn@uic.edu (David P. Nicholls), vtamma2@uic.edu (Venu Tammali)
} 
Multipole Methods [25]) and they are a compelling choice [56]. However, three properties render them non-competitive for the periodic, parametrized problems we consider as compared with the method we advocate here. First, for periodic problems the relevant Green function must be periodized. This is a well-known problem [31] and the slow convergence of the periodization must be accelerated (e.g., with Ewald summation). However, even with such technology, these IE methods demand an additional discretization parameter: The number of terms retained in the approximation of the periodized Green function. Second, for configurations parametrized by the height/slope $\varepsilon$ of the crossed interface, an IE solver must be invoked for every desired value. Finally, the dense, nonsymmetric positive definite systems of linear equations which must be inverted with each simulation.

As an alternative, we advocate here for a "High-Order Perturbation of Surfaces" (HOPS) method, more specifically a Fokas Integral Equation (FIE) reformulation appropriately generalized to the fully three-dimensional vector Maxwell equations [2, 43]. These schemes trace their roots to the low-order calculations of Rayleigh [54] and Rice [57]. Their high-order incarnation has been developed into the Method of Field Expansions (FE) by Bruno \& Reitich [7, 8, 9], the author and Reitich [47, 48, 49], and the author and Malcolm [35, 44]. A closely related algorithm, the Method of Operator Expansions, was developed in parallel by Milder and collaborators [36, 37, 40, 41, 39, 38, 12].

These formulations are particularly compelling as they maintain the advantageous properties of classical Integral Equations formulations (e.g., surface formulation and exact enforcement of far-field conditions) while avoiding the shortcomings listed above. First, as HOPS schemes utilize eigenfunctions of the Laplacian (suitable complex exponentials) on a periodic domain the quasiperiodicity of solutions is "built in" and does not need to be further approximated. Second, since the methods are built upon expansions in the boundary parameter, $\varepsilon$, once the Taylor coefficients are known for the scattering quantities, it is simply a matter of summing these (rather than beginning a new simulation) for any given choice of $\varepsilon$ to recover the returns. Finally, due to the perturbative nature of the scheme, at every perturbation order one need only invert a single, sparse operator corresponding to the flat-interface approximation of the problem.

As we have shown in the context of the Helmholtz equation [2, 43], the approach of Fokas [20] and collaborators (see, e.g., $[1,60,61]$ ) allows one to state simple integral relations for Dirichlet and Neumann data of elliptic boundary value problems which do not involve the fundamental solution. Instead they feature quite smooth kernels related to solutions of the relevant Helmholtz problem meaning that simple quadrature rules (e.g., Nyström's Method [13]) can be brought to bear on the problem. The Fokas approach (known as the "Unified Transform Method") does give rise to dense, poorly conditioned linear systems to be inverted, but one of the authors showed in [43] how this can be significantly ameliorated with a HOPS methodology. In particular, as we shall see, one trades a single dense and ill-conditioned matrix inversion for a sequence (one for each perturbation order retained) of fast, well-conditioned linear solves around the base (flat-interface) geometry. This matrix is of convolution-type which enables rapid solution by the FFT algorithm. Additionally, we find that, for many configurations of interest, only a small number of perturbation orders are required for a solution of high fidelity, resulting in an algorithm of remarkable speed and accuracy.

The authors have recently been made aware of the work of DeSanto and collaborators $[16,17,15$, $3]$ who advanced an approach which is very close to the one presented by the author and Ambrose in [2]. A nice explanation of their approach and how it connects to the formulation of Fokas is given in [10]. In the current contribution, the authors extend this work not only in generalization to multiple layers for the full Maxwell equations, but also through the introduction of a HOPS methodology.

The rest of the paper is organized as follows: In $\S 2$ we briefly recall the equations which govern the interaction of vector electromagnetic waves with a three-dimensional doubly periodic diffraction grating, followed in $\S 2.1$ by a discussion of the classical Rayleigh expansions, efficiencies, and the Reflectivity Map. In $\S 3$ we describe a boundary formulation of these governing equations which allows one to simulate solutions in a rapid and robust fashion. We desribe the details surrounding the interfacial boundary conditions in $\S 3.1$ (the tangential trace), $\S 3.2$ (the tangential curl), and $\S 3.3$ (the divergence free condition). We summarize our surface formulation in $\S 3.4$. In $\S 4$ we describe how a High-Order Perturbation of Surfaces (HOPS) approach can be utilized to perturbatively solve the equations given in $\S 3.4$. We present detailed numerical experiments in $\S 5$, including validation against a Field Expansions (FE) implementation $[9,44]$ in $\S 5.1$, and results of simulations of 
Reflectivity Maps in $\S 5.2$ for configurations involving gratings composed of different metals which generate quite different plasmonic responses.

\section{The governing equations}

Consider a diffraction grating with crossed periodic interface located at

$$
z=g(x, y)
$$

where $z$ is the vertical coordinate and $x$ and $y$ are the lateral coordinates, which delineates two layers

$$
S^{u}:=\{z>g(x, y)\}, \quad S^{w}:=\{z<g(x, y)\} .
$$

The layers are filled with materials having dielectric constants $\left\{\epsilon^{u}, \epsilon^{w}\right\}$, and the permeability of each is equal to $\mu_{0}$, that of the vacuum. In this contribution we consider the genuinely three-dimensional setting where the grating interface is crossed and periodic

$$
g\left(x+d_{1}, y+d_{2}\right)=g(x, y)
$$

see Figure 1.

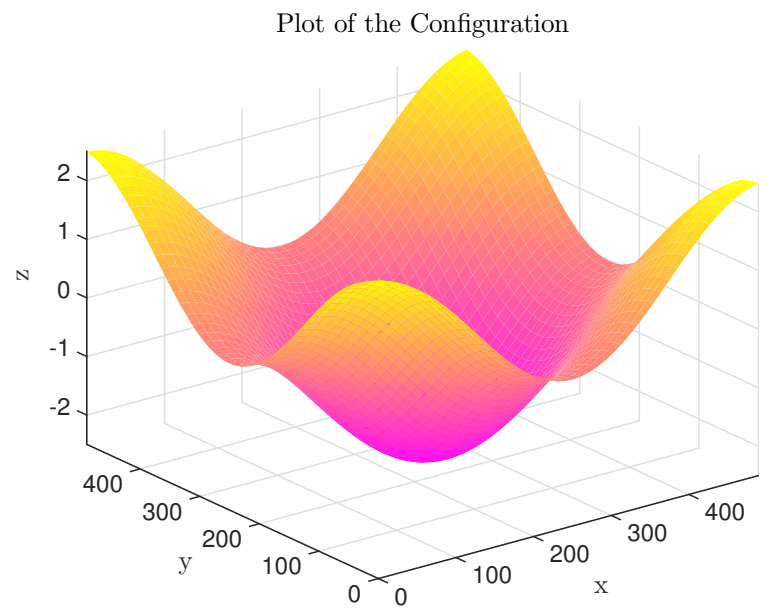

Figure 1: Plot of the configuration with grating interface shaped by $g(x, y)=(\varepsilon / 4)\left(\cos \left(2 \pi x / d_{1}\right)+\cos \left(2 \pi y / d_{2}\right)\right)$, $d_{1}=d_{2}=d=500 \mathrm{~nm}, \varepsilon / d=0.01$ giving $\varepsilon=5 \mathrm{~nm}$.

The structure is illuminated from above by plane-wave incidence of the form

$$
\begin{aligned}
\mathbf{E}^{i n c}(x, y, z) & :=e^{i \omega t} \underline{\mathbf{E}}^{i n c}(x, y, z, t)=\mathbf{A} e^{i(\alpha x+\beta y-\gamma z)} \\
\mathbf{H}^{i n c}(x, y, z) & :=e^{i \omega t} \underline{\mathbf{H}}^{i n c}(x, y, z, t)=\mathbf{B} e^{i(\alpha x+\beta y-\gamma z)},
\end{aligned}
$$

where

$$
\mathbf{A} \cdot \kappa=0, \quad \mathbf{B}=\frac{1}{\omega \mu_{0}} \kappa \times \mathbf{A}, \quad|\mathbf{A}|=|\mathbf{B}|=1,
$$

and $\kappa:=(\alpha, \beta,-\gamma)^{T}$.

The time-harmonic Maxwell equations [29, 51] for the reduced total fields can be written (upon dropping the harmonic factor $\exp (-i \omega t))$ as

$$
\begin{aligned}
\nabla \times \mathbf{E}=i \omega \mu_{0} \mathbf{H}, & \operatorname{div}[\mathbf{E}]=0, \\
\nabla \times \mathbf{H}=-i \omega \epsilon \mathbf{E}, & \operatorname{div}[\mathbf{H}]=0 .
\end{aligned}
$$


All fields satisfy the vector Helmholtz equation, e.g.,

$$
\Delta \mathbf{E}+k^{2} \mathbf{E}=0
$$

with $k^{2}=\omega^{2} \epsilon \mu_{0}$ which encodes the properties of the material and the frequency of radiation into one constant.

We decompose the total fields into incident and scattered components by

$$
\mathbf{E}=\left\{\begin{array}{ll}
\mathbf{E}^{u}+\mathbf{E}^{i n c} & \text { in } S^{u} \\
\mathbf{E}^{w} & \text { in } S^{w}
\end{array}, \quad \mathbf{H}= \begin{cases}\mathbf{H}^{u}+\mathbf{H}^{i n c} & \text { in } S^{u} \\
\mathbf{H}^{w} & \text { in } S^{w}\end{cases}\right.
$$

and note that each of these must satisfy vector Helmholtz equations, e.g.,

$$
\Delta \mathbf{E}^{u}+\left(k^{u}\right)^{2} \mathbf{E}^{u}=0, \quad \text { in } S^{u}
$$

where $\left(k^{u}\right)^{2}:=\omega^{2} \epsilon^{u} \mu_{0}$, and

$$
\alpha^{2}+\beta^{2}+\gamma^{2}=\left(k^{u}\right)^{2} .
$$

At the material interface the fields are coupled by the transmission conditions [29, 51],

$$
\begin{aligned}
& N \times\left[\mathbf{E}^{u}-\mathbf{E}^{w}\right]=\zeta \\
& N \times\left[\mathbf{H}^{u}-\mathbf{H}^{w}\right]=\psi,
\end{aligned}
$$

where $N:=\left(-\partial_{x} g,-\partial_{y} g, 1\right)^{T}$, and, in the present context of plane-wave illumination from above,

$$
\zeta=-N \times\left[\mathbf{E}^{i n c}\right]_{z=g}, \quad \psi=-N \times\left[\mathbf{H}^{i n c}\right]_{z=g} .
$$

In light of Maxwell's equations, (2.1), we can rewrite this in terms of the electric field as

$$
\begin{aligned}
& N \times\left[\mathbf{E}^{u}-\mathbf{E}^{w}\right]_{z=g}=\zeta, \\
& N \times\left[\nabla \times\left[\mathbf{E}^{u}-\mathbf{E}^{w}\right]\right]_{z=g}=\psi,
\end{aligned}
$$

where

$$
\psi=-N \times\left[\nabla \times\left[\mathbf{E}^{i n c}\right]\right]_{z=g} .
$$

We point out that only four of these six boundary conditions are linearly dependent (the $z-$ component of each can be written as a linear combination of the $x$ - and $y$-components, for instance). Clearly it only makes sense to enforce four and we choose the $x$ - and $y$-components of each. To compensate for this "defect" we recall that the electric field is divergence free in the bulk, and must also be so at the interface. Thus we enforce the two additional boundary conditions

$$
\operatorname{div}\left[\mathbf{E}^{u}\right]=\operatorname{div}\left[\mathbf{E}^{w}\right]=0, \quad z=g .
$$

Finally, the periodicity of the grating interfaces implies that the fields are quasiperiodic,

$$
\mathbf{E}^{m}\left(x+d_{1}, y+d_{2}, z\right)=e^{i\left(\alpha d_{1}+\beta d_{2}\right)} \mathbf{E}^{m}(x, y, z), \quad m=u, w,
$$

and we demand that $\mathbf{E}^{u}$ and $\mathbf{E}^{w}$ be outgoing at positive and negative infinity, respectively.

\subsection{The Rayleigh expansions, efficiencies, and the reflectivity map}

Separation of variables gives the Rayleigh expansions [51] which are quasiperiodic, outgoing solutions of (2.2) that we discuss in order to introduce notation. The electric fields can be written

$$
\mathbf{E}^{u}(x, y, z)=\sum_{p=-\infty}^{\infty} \sum_{q=-\infty}^{\infty} \mathbf{a}_{p, q} e^{i \gamma_{p, q}^{u} z} e^{i\left(\alpha_{p} x+\beta_{q} y\right)}
$$


and

$$
\mathbf{E}^{w}(x, y, z)=\sum_{p=-\infty}^{\infty} \sum_{q=-\infty}^{\infty} \mathbf{d}_{p, q} e^{-i \gamma_{p, q}^{w} z} e^{i\left(\alpha_{p} x+\beta_{q} y\right)},
$$

where, for $p, q \in \mathbf{Z}$,

$$
\begin{gathered}
\alpha_{p}:=\alpha+\left(2 \pi / d_{1}\right) p, \quad \beta_{q}:=\beta+\left(2 \pi / d_{2}\right) q, \\
\gamma_{p, q}^{m}:=\left\{\begin{array}{ll}
\sqrt{\left(k^{m}\right)^{2}-\alpha_{p}^{2}-\beta_{q}^{2}} & (p, q) \in \mathcal{U}^{m} \\
i \sqrt{\alpha_{p}^{2}+\beta_{q}^{2}-\left(k^{m}\right)^{2}} & (p, q) \notin \mathcal{U}^{m}
\end{array}, \quad m=u, w,\right.
\end{gathered}
$$

and,

$$
\mathcal{U}^{m}=\left\{p, q \in \mathbf{Z} \mid \alpha_{p}^{2}+\beta_{q}^{2}<\left(k^{m}\right)^{2}\right\}, \quad m=u, w,
$$

which are the "propagating modes" in the upper and lower layers. (We point out that $\mathbf{a}_{p, q}$ and $\mathbf{d}_{p, q}$ are the upward and downward propagating Rayleigh amplitudes.) Quantities of great interest are the efficiencies

$$
\begin{array}{ll}
e_{p, q}^{u}=\left(\gamma_{p, q}^{u} / \gamma\right)\left|\mathbf{a}_{p, q}\right|^{2}, & (p, q) \in \mathcal{U}^{u}, \\
e_{p, q}^{w}=\left(\gamma_{p, q}^{w} / \gamma\right)\left|\mathbf{d}_{p, q}\right|^{2}, & (p, q) \in \mathcal{U}^{w},
\end{array}
$$

and the object of fundamental importance to the design of Surface Plasmon Resonance (SPR) biosensors $[26,28,33,30,55,50]$ is the "Reflectivity Map"

$$
R:=\sum_{(p, q) \in \mathcal{U}^{u}} e_{p, q}^{u} .
$$

If the lower layer is filled with a perfect electric conductor, and a lossless dielectric fills the upper medium, conservation of energy requires that $R=1$. As we shall see this is not the case for a metal (such as gold) in the lower domain and drops in its value to a tenth or even a hundredth are the fundamental phenomenon behind the utility of Surface Plasmon Resonance (SPR) sensors $[52,34,19]$.

\section{Boundary formulation}

We will now demonstrate how the governing equations (2.2) \& (2.3) can be rewritten in terms of surface quantities, more specifically the Dirichlet and (exterior) Neumann traces. For this we define the surface quantities

$$
\begin{aligned}
& \mathbf{U}(x, y):=\mathbf{E}^{u}(x, y, g(x, y)) \\
& \mathbf{W}(x, y):=\mathbf{E}^{w}(x, y, g(x, y)) \\
& \tilde{\mathbf{U}}(x, y):=\left[-\partial_{z} \mathbf{E}^{u}+\left(\partial_{x} g\right) \partial_{x} \mathbf{E}^{u}+\left(\partial_{y} g\right) \partial_{y} \mathbf{E}^{u}\right](x, y, g(x, y)) \\
& \tilde{\mathbf{W}}(x, y):=\left[\partial_{z} \mathbf{E}^{w}-\left(\partial_{x} g\right) \partial_{x} \mathbf{E}^{w}-\left(\partial_{y} g\right) \partial_{y} \mathbf{E}^{w}\right](x, y, g(x, y)),
\end{aligned}
$$

where

$$
\mathbf{U}=\left(\begin{array}{c}
U^{x} \\
U^{y} \\
U^{z}
\end{array}\right), \quad \mathbf{W}=\left(\begin{array}{c}
W^{x} \\
W^{y} \\
W^{z}
\end{array}\right), \quad \tilde{\mathbf{U}}=\left(\begin{array}{c}
\tilde{U}^{x} \\
\tilde{U}^{y} \\
\tilde{U}^{z}
\end{array}\right), \quad \tilde{\mathbf{W}}=\left(\begin{array}{c}
\tilde{W}^{x} \\
\tilde{W}^{y} \\
\tilde{W}^{z}
\end{array}\right)
$$

so

$$
\begin{aligned}
& \{\mathbf{U}, \mathbf{W}, \tilde{\mathbf{U}}, \tilde{\mathbf{W}}\}: \mathbf{R}^{2} \rightarrow \mathbf{R}^{3}, \\
& \left\{U^{j}, W^{j}, \tilde{U}^{j}, \tilde{W}^{j}\right\}: \mathbf{R}^{2} \rightarrow \mathbf{R}, \quad j=x, y, z .
\end{aligned}
$$


As each of the components of $\left\{\mathbf{E}^{u}, \mathbf{E}^{w}\right\}$ is an outgoing, quasiperiodic solution of the Helmholtz equation, (2.2), the Dirichlet and Neumann traces are related by the Fokas Integral Equations (FIE) $[2,43]$

$$
A^{u}\left[\tilde{U}^{j}\right]-R^{u}\left[U^{j}\right]=0, \quad A^{w}\left[\tilde{W}^{j}\right]-R^{w}\left[W^{j}\right]=0, \quad j=x, y, z .
$$

The operators are defined (for $j=x, y, z$ ) by

$$
\begin{aligned}
& A^{u}\left[\tilde{U}^{j}\right]:=\frac{1}{d_{1} d_{2}} \sum_{p=-\infty}^{\infty} \sum_{q=-\infty}^{\infty} \hat{A}_{p, q}^{u}\left[\tilde{U}^{j}\right] e^{i\left(\alpha_{p} x+\beta_{q} y\right)}, \\
& R^{u}\left[U^{j}\right]:=\frac{1}{d_{1} d_{2}} \sum_{p=-\infty}^{\infty} \sum_{q=-\infty}^{\infty} \hat{R}_{p, q}^{u}\left[U^{j}\right] e^{i\left(\alpha_{p} x+\beta_{q} y\right)},
\end{aligned}
$$

and

$$
\begin{aligned}
& A^{w}\left[\tilde{W}^{j}\right]:=\frac{1}{d_{1} d_{2}} \sum_{p=-\infty}^{\infty} \sum_{q=-\infty}^{\infty} \hat{A}_{p, q}^{w}\left[\tilde{W}^{j}\right] e^{i\left(\alpha_{p} x+\beta_{q} y\right)}, \\
& R^{w}\left[W^{j}\right]:=\frac{1}{d_{1} d_{2}} \sum_{p=-\infty}^{\infty} \sum_{q=-\infty}^{\infty} \hat{R}_{p, q}^{w}\left[W^{j}\right] e^{i\left(\alpha_{p} x+\beta_{q} y\right)} .
\end{aligned}
$$

In these

$$
\begin{aligned}
& \hat{A}_{p, q}^{u}[\tilde{U}]:=\int_{0}^{d_{1}} \int_{0}^{d_{2}} e^{i \gamma_{p, q}^{u} g(x, y)} e^{-i\left(\alpha_{p} x+\beta_{q} y\right)} \tilde{U}(x, y) \mathrm{d} x \mathrm{~d} y \\
& \hat{R}_{p, q}^{u}[U]:=\int_{0}^{d_{1}} \int_{0}^{d_{2}} e^{i \gamma_{p, q}^{u} g(x, y)} e^{-i\left(\alpha_{p} x+\beta_{q} y\right)}\left\{\frac{i \alpha_{p} \partial_{x}+i \beta_{q} \partial_{y}+\left(k^{u}\right)^{2}}{i \gamma_{p, q}^{u}}\right\} U(x, y) \mathrm{d} x \mathrm{~d} y \\
& \hat{A}_{p, q}^{w}[\tilde{W}]:=\int_{0}^{d_{1}} \int_{0}^{d_{2}} e^{-i \gamma_{p, q}^{w} g(x, y)} e^{-i\left(\alpha_{p} x+\beta_{q} y\right)} \tilde{W}(x, y) \mathrm{d} x \mathrm{~d} y \\
& \hat{R}_{p, q}^{w}[W]:=\int_{0}^{d_{1}} \int_{0}^{d_{2}} e^{-i \gamma_{p, q}^{w} g(x, y)} e^{-i\left(\alpha_{p} x+\beta_{q} y\right)}\left\{\frac{i \alpha_{p} \partial_{x}+i \beta_{q} \partial_{y}+\left(k^{w}\right)^{2}}{i \gamma_{p, q}^{w}}\right\} W(x, y) \mathrm{d} x \mathrm{~d} y .
\end{aligned}
$$

\subsection{Tangential trace}

Regarding the boundary conditions, we begin with the trace of the tangential component of a vector field. For this we have

$$
N \times \mathbf{U}=S \mathbf{U}
$$

where

$$
S:=\left(\begin{array}{ccc}
0 & -1 & -\left(\partial_{y} g\right) \\
1 & 0 & \left(\partial_{x} g\right) \\
\left(\partial_{y} g\right) & -\left(\partial_{x} g\right) & 0
\end{array}\right) .
$$

We further require the $(x, y)$-projection operator

$$
P_{x, y} \mathbf{U}=P_{x, y}\left(\begin{array}{l}
U^{x} \\
U^{y} \\
U^{z}
\end{array}\right)=\left(\begin{array}{l}
U^{x} \\
U^{y}
\end{array}\right),
$$

so that

$$
P_{x, y}:=\left(\begin{array}{ccc}
1 & 0 & 0 \\
0 & 1 & 0
\end{array}\right)
$$

This gives

$$
S_{x, y}:=P_{x, y} S=\left(\begin{array}{ccc}
S^{x x} & S^{x y} & S^{x z} \\
S^{y x} & S^{y y} & S^{y z}
\end{array}\right)
$$

where

$$
S^{x x}=0, \quad S^{x y}=-1, \quad S^{x z}=-\left(\partial_{y} g\right), \quad S^{y x}=1, \quad S^{y y}=0, \quad S^{y z}=\left(\partial_{x} g\right) .
$$

Thus, the two ( $x$ - and $y$-components of the) Dirichlet boundary conditions, (2.3a), read

$$
S_{x, y} \mathbf{U}-S_{x, y} \mathbf{W}=P_{x, y} \zeta .
$$




\subsection{Tangential curl}

We recall that the curl of $\mathbf{E}^{m}$ is given by

$$
\nabla \times \mathbf{E}^{m}=\left(\begin{array}{c}
\partial_{y} E^{m, z}-\partial_{z} E^{m, y} \\
\partial_{z} E^{m, x}-\partial_{x} E^{m, z} \\
\partial_{x} E^{m, y}-\partial_{y} E^{m, x}
\end{array}\right), \quad m=u, w .
$$

We now change to the surface variables $\{\mathbf{U}, \mathbf{W}\}$ with tangential derivatives $\left\{\left(\partial_{x} \mathbf{U}, \partial_{y} \mathbf{U}\right),\left(\partial_{x} \mathbf{W}, \partial_{y} \mathbf{W}\right)\right\}$, and normal derivatives, $\{\tilde{\mathbf{U}}, \tilde{\mathbf{W}}\}$. The chain rule gives

$$
\begin{aligned}
& \partial_{x} \mathbf{U}(x, y)=\left[\partial_{x} \mathbf{E}^{u}+\left(\partial_{x} g\right) \partial_{z} \mathbf{E}^{u}\right](x, y, g(x, y)), \\
& \partial_{y} \mathbf{U}(x, y)=\left[\partial_{y} \mathbf{E}^{u}+\left(\partial_{y} g\right) \partial_{z} \mathbf{E}^{u}\right](x, y, g(x, y)), \\
& \partial_{x} \mathbf{W}(x, y)=\left[\partial_{x} \mathbf{E}^{w}+\left(\partial_{x} g\right) \partial_{z} \mathbf{E}^{w}\right](x, y, g(x, y)), \\
& \partial_{y} \mathbf{W}(x, y)=\left[\partial_{y} \mathbf{E}^{w}+\left(\partial_{y} g\right) \partial_{z} \mathbf{E}^{w}\right](x, y, g(x, y)),
\end{aligned}
$$

while (3.1) gives formulas for $\tilde{\mathbf{U}}$ and $\tilde{\mathbf{W}}$. It is not difficult to see that

$$
\begin{aligned}
& |N|^{2} \partial_{x} \mathbf{E}^{u}=\left(|N|^{2}-\left(\partial_{x} g\right)^{2}\right) \partial_{x} \mathbf{U}-\left(\partial_{x} g\right)\left(\partial_{y} g\right) \partial_{y} \mathbf{U}+\left(\partial_{x} g\right) \tilde{\mathbf{U}} \\
& |N|^{2} \partial_{y} \mathbf{E}^{u}=-\left(\partial_{x} g\right)\left(\partial_{y} g\right) \partial_{x} \mathbf{U}+\left(|N|^{2}-\left(\partial_{y} g\right)^{2}\right) \partial_{y} \mathbf{U}+\left(\partial_{y} g\right) \tilde{\mathbf{U}} \\
& |N|^{2} \partial_{z} \mathbf{E}^{u}=\left(\partial_{x} g\right) \partial_{x} \mathbf{U}+\left(\partial_{y} g\right) \partial_{y} \mathbf{U}-\tilde{\mathbf{U}}
\end{aligned}
$$

and

$$
\begin{aligned}
& |N|^{2} \partial_{x} \mathbf{E}^{w}=\left(|N|^{2}-\left(\partial_{x} g\right)^{2}\right) \partial_{x} \mathbf{W}-\left(\partial_{x} g\right)\left(\partial_{y} g\right) \partial_{y} \mathbf{W}-\left(\partial_{x} g\right) \tilde{\mathbf{W}} \\
& |N|^{2} \partial_{y} \mathbf{E}^{w}=-\left(\partial_{x} g\right)\left(\partial_{y} g\right) \partial_{x} \mathbf{W}+\left(|N|^{2}-\left(\partial_{y} g\right)^{2}\right) \partial_{y} \mathbf{W}-\left(\partial_{y} g\right) \tilde{\mathbf{W}} \\
& |N|^{2} \partial_{z} \mathbf{E}^{w}=\left(\partial_{x} g\right) \partial_{x} \mathbf{W}+\left(\partial_{y} g\right) \partial_{y} \mathbf{W}+\tilde{\mathbf{W}}
\end{aligned}
$$

where

$$
|N|^{2}=\left(\partial_{x} g\right)^{2}+\left(\partial_{y} g\right)^{2}+1
$$

In light of this inconvenient pre-factor, regarding the tangential curl boundary condition, (2.3b), we premultiply by $|N|^{2}$ and enforce the equivalent equation

$$
N \times\left[|N|^{2} \nabla \times\left[\mathbf{E}^{u}-\mathbf{E}^{w}\right]\right]_{z=g}=|N|^{2} \psi .
$$

Regarding the curl of $\mathbf{E}^{u}$ we now proceed deliberately, beginning with the $x$-component

$$
\begin{aligned}
|N|^{2}\left(\partial_{y} E^{u, z}-\partial_{z} E^{u, y}\right)= & \left\{-\left(\partial_{x} g\right)\left(\partial_{y} g\right) \partial_{x} U^{z}+\left(|N|^{2}-\left(\partial_{y} g\right)^{2}\right) \partial_{y} U^{z}+\left(\partial_{y} g\right) \tilde{U}^{z}\right\} \\
& -\left\{\left(\partial_{x} g\right) \partial_{x} U^{y}+\left(\partial_{y} g\right) \partial_{x} U^{y}-\tilde{U}^{y}\right\} \\
= & \tilde{C}^{u, x x} \tilde{U}^{x}+\tilde{C}^{u, x y} \tilde{U}^{y}+\tilde{C}^{u, x z} \tilde{U}^{z}+C^{u, x x} U^{x}+C^{u, x y} U^{y}+C^{u, x z} U^{z},
\end{aligned}
$$

where

$$
\begin{aligned}
& \tilde{C}^{u, x x}=0, \\
& \tilde{C}^{u, x y}=1, \\
& \tilde{C}^{u, x z}=\left(\partial_{y} g\right), \\
& C^{u, x x}=0, \\
& C^{u, x y}=-\left(\partial_{x} g\right) \partial_{x}-\left(\partial_{y} g\right) \partial_{y}, \\
& C^{u, x z}=-\left(\partial_{x} g\right)\left(\partial_{y} g\right) \partial_{x}+\left(|N|^{2}-\left(\partial_{y} g\right)^{2}\right) \partial_{y} .
\end{aligned}
$$


Continuing, for the $y$-component,

$$
\begin{aligned}
|N|^{2}\left(\partial_{z} E^{u, x}-\partial_{x} E^{u, z}\right)= & \left\{\left(\partial_{x} g\right) \partial_{x} U^{x}+\left(\partial_{y} g\right) \partial_{y} U^{x}-\tilde{U}^{x}\right\} \\
& -\left\{\left(|N|^{2}-\left(\partial_{x} g\right)^{2}\right) \partial_{x} U^{z}-\left(\partial_{x} g\right)\left(\partial_{y} g\right) \partial_{y} U^{z}+\left(\partial_{x} g\right) \tilde{U}^{z}\right\} \\
= & \tilde{C}^{u, y x} \tilde{U}^{x}+\tilde{C}^{u, y y} \tilde{U}^{y}+\tilde{C}^{u, y z} \tilde{U}^{z}+C^{u, y x} U^{x}+C^{u, y y} U^{y}+C^{u, y z} U^{z},
\end{aligned}
$$

where

$$
\begin{aligned}
& \tilde{C}^{u, y x}=-1, \\
& \tilde{C}^{u, y y}=0 \\
& \tilde{C}^{u, y z}=-\left(\partial_{x} g\right), \\
& C^{u, y x}=\left(\partial_{x} g\right) \partial_{x}+\left(\partial_{y} g\right) \partial_{y}, \\
& C^{u, y y}=0 \\
& C^{u, y z}=-\left(|N|^{2}-\left(\partial_{x} g\right)^{2}\right) \partial_{x}+\left(\partial_{x} g\right)\left(\partial_{y} g\right) \partial_{y} .
\end{aligned}
$$

Finally, for the $z$-component,

$$
\begin{aligned}
|N|^{2}\left(\partial_{x} E^{u, y}-\partial_{y} E^{u, x}\right)= & \left\{\left(|N|^{2}-\left(\partial_{x} g\right)^{2}\right) \partial_{x} U^{y}-\left(\partial_{x} g\right)\left(\partial_{y} g\right) \partial_{y} U^{y}+\left(\partial_{x} g\right) \tilde{U}^{y}\right\} \\
& -\left\{-\left(\partial_{x} g\right)\left(\partial_{y} g\right) \partial_{x} U^{x}+\left(|N|^{2}-\left(\partial_{y} g\right)^{2}\right) \partial_{y} U^{x}+\left(\partial_{y} g\right) \tilde{U}^{x}\right\} \\
= & \tilde{C}^{u, z x} \tilde{U}^{x}+\tilde{C}^{u, z y} \tilde{U}^{y}+\tilde{C}^{u, z z} \tilde{U}^{z}+C^{u, z x} U^{x}+C^{u, z y} U^{y}+C^{u, z z} U^{z},
\end{aligned}
$$

where

$$
\begin{aligned}
& \tilde{C}^{u, z x}=-\left(\partial_{y} g\right), \\
& \tilde{C}^{u, z y}=\left(\partial_{x} g\right), \\
& \tilde{C}^{u, z z}=0 \\
& C^{u, z x}=\left(\partial_{x} g\right)\left(\partial_{y} g\right) \partial_{x}-\left(|N|^{2}-\left(\partial_{y} g\right)^{2}\right) \partial_{y}, \\
& C^{u, z y}=\left(|N|^{2}-\left(\partial_{x} g\right)^{2}\right) \partial_{x}-\left(\partial_{x} g\right)\left(\partial_{y} g\right) \partial_{y}, \\
& C^{u, z z}=0 .
\end{aligned}
$$

Defining

$$
\tilde{C}^{u}=\left(\begin{array}{ccc}
\tilde{C}^{u, x x} & \tilde{C}^{u, x y} & \tilde{C}^{u, x z} \\
\tilde{C}^{u, y x} & \tilde{C}^{u, y y} & \tilde{C}^{u, y z} \\
\tilde{C}^{u, z x} & \tilde{C}^{u, z y} & \tilde{C}^{u, z z}
\end{array}\right), \quad C^{u}=\left(\begin{array}{lll}
C^{u, x x} & C^{u, x y} & C^{u, x z} \\
C^{u, y x} & C^{u, y y} & C^{u, y z} \\
C^{u, z x} & C^{u, z y} & C^{u, z z}
\end{array}\right)
$$

we have

$$
|N|^{2} \nabla \times \mathbf{E}^{u}=\left(\begin{array}{ll}
\tilde{C}^{u} & C^{u}
\end{array}\right)\left(\begin{array}{c}
\tilde{\mathbf{U}} \\
\mathbf{U}
\end{array}\right) .
$$

Again considering that we only wish to enforce these for the $x$ - and $y$-components, we define

$$
\begin{aligned}
& C_{x, y}^{u}:=S_{x, y} C^{u}=\left(\begin{array}{ccc}
-C^{u, y x}-\left(\partial_{y} g\right) C^{u, z x} & -C^{u, y y}-\left(\partial_{y} g\right) C^{u, z y} & -C^{u, y z}-\left(\partial_{y} g\right) C^{u, z z} \\
C^{u, x x}+\left(\partial_{x} g\right) C^{u, z x} & C^{u, x y}+\left(\partial_{x} g\right) C^{u, z y} & C^{u, x z}+\left(\partial_{x} g\right) C^{u, z z}
\end{array}\right) \\
& \tilde{C}_{x, y}^{u}:=S_{x, y} \tilde{C}^{u}=\left(\begin{array}{ccc}
-\tilde{C}^{u, y x}-\left(\partial_{y} g\right) \tilde{C}^{u, z x} & -\tilde{C}^{u, y y}-\left(\partial_{y} g\right) \tilde{C}^{u, z y} & -\tilde{C}^{u, y z}-\left(\partial_{y} g\right) \tilde{C}^{u, z z} \\
\tilde{C}^{u, x x}+\left(\partial_{x} g\right) \tilde{C}^{u, z x} & \tilde{C}^{u, x y}+\left(\partial_{x} g\right) \tilde{C}^{u, z y} & \tilde{C}^{u, x z}+\left(\partial_{x} g\right) \tilde{C}^{u, z z}
\end{array}\right) .
\end{aligned}
$$

In an analogous manner one can derive for the curl of $\mathbf{E}^{w}$ that

$$
|N|^{2} \nabla \times \mathbf{E}^{u}=\left(\begin{array}{ll}
\tilde{C}^{w} & C^{w}
\end{array}\right)\left(\begin{array}{l}
\tilde{\mathbf{U}} \\
\mathbf{U}
\end{array}\right) .
$$


where

$$
\tilde{C}^{w}=\left(\begin{array}{lll}
\tilde{C}^{w, x x} & \tilde{C}^{w, x y} & \tilde{C}^{w, x z} \\
\tilde{C}^{w, y x} & \tilde{C}^{w, y y} & \tilde{C}^{w, y z} \\
\tilde{C}^{w, z x} & \tilde{C}^{w, z y} & \tilde{C}^{w, z z}
\end{array}\right), \quad C^{w}=\left(\begin{array}{lll}
C^{w, x x} & C^{w, x y} & C^{w, x z} \\
C^{w, y x} & C^{w, y y} & C^{w, y z} \\
C^{w, z x} & C^{w, z y} & C^{w, z z}
\end{array}\right)
$$

As before we define

$$
\begin{aligned}
& C_{x, y}^{w}:=S_{x, y} C^{w}=\left(\begin{array}{ccc}
-C^{w, y x}-\left(\partial_{y} g\right) C^{w, z x} & -C^{w, y y}-\left(\partial_{y} g\right) C^{w, z y} & -C^{w, y z}-\left(\partial_{y} g\right) C^{w, z z} \\
C^{w, x x}+\left(\partial_{x} g\right) C^{w, z x} & C^{w, x y}+\left(\partial_{x} g\right) C^{w, z y} & C^{w, x z}+\left(\partial_{x} g\right) C^{w, z z}
\end{array}\right) \\
& \tilde{C}_{x, y}^{w}:=S_{x, y} \tilde{C}^{w}=\left(\begin{array}{ccc}
-\tilde{C}^{w, y x}-\left(\partial_{y} g\right) \tilde{C}^{w, z x} & -\tilde{C}^{w, y y}-\left(\partial_{y} g\right) \tilde{C}^{w, z y} & -\tilde{C}^{w, y z}-\left(\partial_{y} g\right) \tilde{C}^{w, z z} \\
\tilde{C}^{w, x x}+\left(\partial_{x} g\right) \tilde{C}^{w, z x} & \tilde{C}^{w, x y}+\left(\partial_{x} g\right) \tilde{C}^{w, z y} & \tilde{C}^{w, x z}+\left(\partial_{x} g\right) \tilde{C}^{w, z z}
\end{array}\right),
\end{aligned}
$$

so that the ( $x$ - and $y$-components of the) two Neumann boundary conditions, (3.5), read

$$
\tilde{C}_{x, y}^{u} \tilde{\mathbf{U}}+C_{x, y}^{u} \mathbf{U}-\tilde{C}_{x, y}^{w} \tilde{\mathbf{W}}-C_{x, y}^{w} \mathbf{W}=P_{x, y}|N|^{2} \psi
$$

The entries of these operators can be shown to be

$$
\begin{aligned}
& \tilde{C}^{w, x x}=0, \\
& \tilde{C}^{w, x y}=-1, \\
& \tilde{C}^{w, x z}=-\left(\partial_{y} g\right), \\
& C^{w, x x}=0, \\
& C^{w, x y}=-\left(\partial_{x} g\right) \partial_{x}-\left(\partial_{y} g\right) \partial_{y}, \\
& C^{w, x z}=-\left(\partial_{x} g\right)\left(\partial_{y} g\right) \partial_{x}+\left(|N|^{2}-\left(\partial_{y} g\right)^{2}\right) \partial_{y},
\end{aligned}
$$

and

$$
\begin{aligned}
& \tilde{C}^{w, y x}=1 \\
& \tilde{C}^{w, y y}=0 \\
& \tilde{C}^{w, y z}=\left(\partial_{x} g\right) \\
& C^{w, y x}=\left(\partial_{x} g\right) \partial_{x}+\left(\partial_{y} g\right) \partial_{y} \\
& C^{w, y y}=0 \\
& C^{w, y z}=-\left(|N|^{2}-\left(\partial_{y} g\right)^{2}\right) \partial_{x}+\left(\partial_{x} g\right)\left(\partial_{y} g\right) \partial_{y}
\end{aligned}
$$

and

$$
\begin{aligned}
& \tilde{C}^{w, z x}=\left(\partial_{y} g\right), \\
& \tilde{C}^{w, z y}=-\left(\partial_{x} g\right), \\
& \tilde{C}^{w, z z}=0, \\
& C^{w, z x}=\left(\partial_{x} g\right)\left(\partial_{y} g\right) \partial_{x}-\left(|N|^{2}-\left(\partial_{y} g\right)^{2}\right) \partial_{y}, \\
& C^{w, z y}=\left(|N|^{2}-\left(\partial_{x} g\right)^{2}\right) \partial_{x}-\left(\partial_{x} g\right)\left(\partial_{y} g\right) \partial_{y}, \\
& C^{w, z z}=0 .
\end{aligned}
$$




\subsection{Divergence-free conditions}

Finally, we enforce the divergence-free condition (again premultiplied by the factor $|N|^{2}$ ) in the new variables

$$
\begin{aligned}
|N|^{2} \operatorname{div}\left[\mathbf{E}^{u}\right]= & |N|^{2}\left(\partial_{x} E^{u, x}+\partial_{y} E^{u, y}+\partial_{z} E^{u, z}\right) \\
= & \left(|N|^{2}-\left(\partial_{x} g\right)^{2}\right) \partial_{x} U^{x}-\left(\partial_{x} g\right)\left(\partial_{y} g\right) \partial_{y} U^{x}+\left(\partial_{x} g\right) \tilde{U}^{x} \\
& +-\left(\partial_{x} g\right)\left(\partial_{y} g\right) \partial_{x} U^{y}+\left(|N|^{2}-\left(\partial_{y} g\right)^{2}\right) \partial_{y} U^{y}+\left(\partial_{y} g\right) \tilde{U}^{y} \\
& +\left(\partial_{x} g\right) \partial_{x} U^{z}+\left(\partial_{y} g\right) \partial_{y} U^{z}-\tilde{U}^{z} \\
= & \tilde{D}^{u, x} \tilde{U}^{x}+\tilde{D}^{u, y} \tilde{U}^{y}+\tilde{D}^{u, z} \tilde{U}^{z}+D^{u, x} U^{x}+D^{u, y} U^{y}+D^{u, z} U^{z},
\end{aligned}
$$

where,

$$
\begin{aligned}
& \tilde{D}^{u, x}=\left(\partial_{x} g\right), \\
& \tilde{D}^{u, y}=\left(\partial_{y} g\right), \\
& \tilde{D}^{u, z}=-1, \\
& D^{u, x}=\left(|N|^{2}-\left(\partial_{x} g\right)^{2}\right) \partial_{x}-\left(\partial_{x} g\right)\left(\partial_{y} g\right) \partial_{y}, \\
& D^{u, y}=-\left(\partial_{x} g\right)\left(\partial_{y} g\right) \partial_{x}+\left(|N|^{2}-\left(\partial_{y} g\right)^{2}\right) \partial_{y}, \\
& D^{u, z}=\left(\partial_{x} g\right) \partial_{x}+\left(\partial_{y} g\right) \partial_{y} .
\end{aligned}
$$

In a similar manner,

$$
\begin{aligned}
|N|^{2} \operatorname{div}\left[\mathbf{E}^{w}\right] & =|N|^{2}\left(\partial_{x} E^{w, x}+\partial_{y} E^{w, y}+\partial_{z} E^{w, z}\right) \\
& =\tilde{D}^{w, x} \tilde{W}^{x}+\tilde{D}^{w, y} \tilde{W}^{y}+\tilde{D}^{w, z} \tilde{W}^{z}+D^{w, x} W^{x}+D^{w, y} W^{y}+D^{w, z} W^{z},
\end{aligned}
$$

where

$$
\begin{aligned}
\tilde{D}^{w, x} & =-\left(\partial_{x} g\right), \\
\tilde{D}^{w, y} & =-\left(\partial_{y} g\right), \\
\tilde{D}^{w, z} & =1, \\
D^{w, x} & =\left(|N|^{2}-\left(\partial_{x} g\right)^{2}\right) \partial_{x}-\left(\partial_{x} g\right)\left(\partial_{y} g\right) \partial_{y}, \\
D^{w, y} & =-\left(\partial_{x} g\right)\left(\partial_{y} g\right) \partial_{x}+\left(|N|^{2}-\left(\partial_{y} g\right)^{2}\right) \partial_{y}, \\
D^{w, z} & =\left(\partial_{x} g\right) \partial_{x}+\left(\partial_{y} g\right) \partial_{y} .
\end{aligned}
$$

If we define

$$
\begin{aligned}
\tilde{D}^{u}:=\left(\begin{array}{llll}
\tilde{D}^{u, x} & \tilde{D}^{u, y} & \tilde{D}^{u, z}
\end{array}\right), & D^{u}:=\left(\begin{array}{llll}
D^{u, x} & D^{u, y} & D^{u, z}
\end{array}\right), \\
\tilde{D}^{w}:=\left(\begin{array}{llll}
\tilde{D}^{w, x} & \tilde{D}^{w, y} & \tilde{D}^{w, z}
\end{array}\right), & D^{w}:=\left(\begin{array}{lll}
D^{w, x} & D^{w, y} & D^{w, z}
\end{array}\right),
\end{aligned}
$$

then the two divergence-free conditions read

$$
\left(\begin{array}{ll}
\tilde{D}^{u} & D^{u}
\end{array}\right)\left(\begin{array}{c}
\tilde{\mathbf{U}} \\
\mathbf{U}
\end{array}\right)=0, \quad\left(\begin{array}{ll}
\tilde{D}^{w} & D^{w}
\end{array}\right)\left(\begin{array}{c}
\tilde{\mathbf{W}} \\
\mathbf{W}
\end{array}\right)=0
$$

\subsection{Surface equations}

Summarizing all of these developments, we find that we must solve (3.2), (3.4), (3.9), and (3.15) which we state abstractly as

$$
M v=b
$$


where

$$
M=\left(\begin{array}{cc}
\tilde{M}_{B C} & M_{B C} \\
\tilde{M}_{D N} & M_{D N}
\end{array}\right), \quad v=\left(\begin{array}{c}
\tilde{\mathbf{U}} \\
\tilde{\mathbf{W}} \\
\mathbf{U} \\
\mathbf{W}
\end{array}\right), \quad b=\left(\begin{array}{l}
b_{B C} \\
b_{D N}
\end{array}\right)
$$

In these

$$
\tilde{M}_{B C}=\left(\begin{array}{cccccc}
0 & 0 & 0 & 0 & 0 & 0 \\
0 & 0 & 0 & 0 & 0 & 0 \\
\tilde{C}^{u, x x} & \tilde{C}^{u, x y} & \tilde{C}^{u, x z} & -\tilde{C}^{w, x x} & -\tilde{C}^{w, x y} & -\tilde{C}^{w, x z} \\
\tilde{C}^{u, y x} & \tilde{C}^{u, y y} & \tilde{C}^{u, y z} & -\tilde{C}^{w, y x} & -\tilde{C}^{w, y y} & -\tilde{C}^{w, y z} \\
\tilde{D}^{u, x} & \tilde{D}^{u, y} & \tilde{D}^{u, z} & 0 & 0 & 0 \\
0 & 0 & 0 & \tilde{D}^{w, x} & \tilde{D}^{w, y} & \tilde{D}^{w, z}
\end{array}\right)
$$

and

$$
M_{B C}=\left(\begin{array}{cccccc}
S^{x x} & S^{x y} & S^{x z} & -S^{x x} & -S^{x y} & -S^{x z} \\
S^{y x} & S^{y y} & S^{y z} & -S^{y x} & -S^{y y} & -S^{y z} \\
C^{u, x x} & C^{u, x y} & C^{u, x z} & -C^{w, x x} & -C^{w, x y} & -C^{w, x z} \\
C^{u, y x} & C^{u, y y} & C^{u, y z} & -C^{w, y x} & -C^{w, y y} & -C^{w, y z} \\
D^{u, x} & D^{u, y} & D^{u, z} & 0 & 0 & 0 \\
0 & 0 & 0 & D^{w, x} & D^{w, y} & D^{w, z}
\end{array}\right),
$$

and

$$
\tilde{M}_{D N}=\left(\begin{array}{cccccc}
A^{u} & 0 & 0 & 0 & 0 & 0 \\
0 & A^{u} & 0 & 0 & 0 & 0 \\
0 & 0 & A^{u} & 0 & 0 & 0 \\
0 & 0 & 0 & A^{w} & 0 & 0 \\
0 & 0 & 0 & 0 & A^{w} & 0 \\
0 & 0 & 0 & 0 & 0 & A^{w}
\end{array}\right)
$$

and

$$
M_{D N}=\left(\begin{array}{cccccc}
-R^{u} & 0 & 0 & 0 & 0 & 0 \\
0 & -R^{u} & 0 & 0 & 0 & 0 \\
0 & 0 & -R^{u} & 0 & 0 & 0 \\
0 & 0 & 0 & -R^{w} & 0 & 0 \\
0 & 0 & 0 & 0 & -R^{w} & 0 \\
0 & 0 & 0 & 0 & 0 & -R^{w}
\end{array}\right)
$$

and

$$
b_{B C}=\left(\begin{array}{c}
-\zeta^{y}-\left(\partial_{y} g\right) \zeta^{z} \\
\zeta^{x}+\left(\partial_{x} g\right) \zeta^{z} \\
-\psi^{y}-\left(\partial_{y} g\right) \psi^{z} \\
\psi^{x}+\left(\partial_{x} g\right) \psi^{z} \\
0 \\
0
\end{array}\right), \quad b_{D N}=\left(\begin{array}{l}
0 \\
0 \\
0 \\
0 \\
0 \\
0
\end{array}\right)
$$

\section{A high-order perturbation of surfaces (HOPS) approach}

Our High-Order Perturbation of Surfaces (HOPS) methodology for solving (3.16) is a straightforward application of regular perturbation theory under the assumption $g(x)=\varepsilon f(x)$. It can be shown that not only are the (known) linear operator $M=M(g)=M(\varepsilon f)$ and inhomogeneity $b=b(g)=b(\varepsilon f)$ analytic functions of $\varepsilon$ for $f$ sufficiently smooth (e.g., $C^{2}, C^{1+\alpha}$, or even Lipschitz), so is our unknown $v=v(g)=v(\varepsilon f)[45,46,48]$. Therefore we can make the strongly convergent expansions

$$
\{M, v, b\}=\{M, v, b\}(g)=\{M, v, b\}(\varepsilon f)=\sum_{n=0}^{\infty}\left\{M_{n}, v_{n}, b_{n}\right\}(f) \varepsilon^{n} .
$$


Insertion of these into (3.16) followed by equating at order $\varepsilon^{n}$ yields

$$
M_{0} v_{n}=b_{n}-\sum_{\ell=0}^{n-1} M_{n-\ell} v_{\ell} .
$$

Note that at each perturbation order, while one must apply the operators $M_{n-\ell}$, one need only invert the flat-interface operator $M_{0}$ (repeatedly). Once this has been accomplished for a range of orders $0 \leq n \leq N$ (delivering $v_{n}$ ) one can form an approximate solution

$$
v^{N}(x, y ; \varepsilon):=\sum_{n=0}^{N} v_{n}(x, y) \varepsilon^{n} .
$$

All that remains is to specify $M_{n}$ and $b_{n}$. These come from (3.17) and it is clear that this will mandate expansions

$$
\begin{aligned}
\left\{\tilde{M}_{B C}, M_{B C}, \tilde{M}_{D N}, M_{D N}, b_{B C}, b_{D N}\right\} & (\varepsilon f) \\
& =\sum_{n=0}^{\infty}\left\{\tilde{M}_{B C, n}, M_{B C, n}, \tilde{M}_{D N, n}, M_{D N, n}, b_{B C, n}, b_{D N, n}\right\}(f) \varepsilon^{n}
\end{aligned}
$$

This, in turn, requires forms for

$$
\begin{aligned}
\left\{S^{r s}, \tilde{C}^{m, r s}, C^{m, r s}, \tilde{D}^{m, r},\right. & \left.D^{m, r}, A^{u}, A^{w}, R^{u}, R^{w}, \zeta^{r}, \psi^{r}\right\} \\
& =\sum_{n=0}^{\infty}\left\{S_{n}^{r s}, \tilde{C}_{n}^{m, r s}, C_{n}^{m, r s}, \tilde{D}_{n}^{m, r}, D_{n}^{m, r}, A_{n}^{u}, A_{n}^{w}, R_{n}^{u}, R_{n}^{w}, \zeta_{n}^{r}, \psi_{n}^{r}\right\}(f) \varepsilon^{n}
\end{aligned}
$$

for $m \in\{u, w\}$ and $r, s \in\{x, y, z\}$. All of these are easily derived, and forms for $\left\{A_{n}^{u}, A_{n}^{w}, R_{n}^{u}, R_{n}^{w}, \zeta_{n}, \psi_{n}\right\}$ appear in one of the author's previous work [43]. We presently specify the remainder.

From (3.3) we have the non-zero components

$$
S_{0}^{x y}=-1, \quad S_{1}^{x z}=-\left(\partial_{y} f\right), \quad S_{0}^{y x}=1, \quad S_{1}^{y z}=\left(\partial_{x} f\right) .
$$

Equations (3.6), (3.7), and (3.8) give the non-zero members

$$
\begin{gathered}
\tilde{C}_{0}^{u, x y}=1, \quad \tilde{C}_{1}^{u, x z}=\left(\partial_{y} f\right), \\
C_{1}^{u, x y}=-\left(\partial_{x} f\right) \partial_{x}-\left(\partial_{y} f\right) \partial_{y}, \quad C_{0}^{u, x z}=\partial_{y}, \quad C_{2}^{u, x z}=-\left(\partial_{x} f\right)\left(\partial_{y} f\right) \partial_{x}+\left(\partial_{x} f\right)^{2} \partial_{y},
\end{gathered}
$$

and

$$
\begin{gathered}
\tilde{C}_{0}^{u, y x}=-1, \quad \tilde{C}_{1}^{u, y z}=-\left(\partial_{x} f\right), \\
C_{1}^{u, y x}=\left(\partial_{x} f\right) \partial_{x}+\left(\partial_{y} f\right) \partial_{y}, \quad C_{0}^{u, y z}=-\partial_{x}, \quad C_{2}^{u, y z}=-\left(\partial_{y} f\right)^{2} \partial_{x}+\left(\partial_{x} f\right)\left(\partial_{y} f\right) \partial_{y},
\end{gathered}
$$

and

$$
\begin{gathered}
\tilde{C}_{1}^{u, z x}=-\left(\partial_{y} f\right), \quad \tilde{C}_{1}^{u, z y}=\left(\partial_{x} f\right) \\
C_{0}^{u, z x}=-\partial_{y}, \quad C_{2}^{u, z x}=\left(\partial_{x} f\right)\left(\partial_{y} f\right) \partial_{x}-\left(\partial_{x} f\right)^{2} \partial_{y} \\
C_{0}^{u, z y}=\partial_{x}, \quad C_{2}^{u, z y}=\left(\partial_{y} f\right)^{2} \partial_{x}-\left(\partial_{x} f\right)\left(\partial_{y} f\right) \partial_{y} .
\end{gathered}
$$

Similarly, (3.10), (3.11), and (3.12) deliver

$$
\begin{gathered}
\tilde{C}_{0}^{w, x y}=-1, \quad \tilde{C}_{1}^{w, x z}=-\left(\partial_{y} f\right), \\
C_{1}^{w, x y}=-\left(\partial_{x} f\right) \partial_{x}-\left(\partial_{y} f\right) \partial_{y}, \quad C_{0}^{w, x z}=\partial_{y}, \quad C_{2}^{w, x z}=-\left(\partial_{x} f\right)\left(\partial_{y} f\right) \partial_{x}+\left(\partial_{x} f\right)^{2} \partial_{y},
\end{gathered}
$$


and

$$
\begin{gathered}
\tilde{C}_{0}^{w, y x}=1, \quad \tilde{C}_{1}^{w, y z}=\left(\partial_{x} f\right), \\
C_{1}^{w, y x}=\left(\partial_{x} f\right) \partial_{x}+\left(\partial_{y} f\right) \partial_{y}, \quad C_{0}^{w, y z}=-\partial_{x}, \quad C_{2}^{w, y z}=-\left(\partial_{y} f\right)^{2} \partial_{x}+\left(\partial_{x} f\right)\left(\partial_{y} f\right) \partial_{y},
\end{gathered}
$$

and

$$
\begin{gathered}
\tilde{C}_{1}^{w, z x}=\left(\partial_{y} f\right), \quad \tilde{C}_{1}^{w, z y}=-\left(\partial_{x} f\right), \\
C_{0}^{w, z x}=-\partial_{y}, \quad C_{2}^{w, z x}=\left(\partial_{x} f\right)\left(\partial_{y} f\right) \partial_{x}-\left(\partial_{x} f\right)^{2} \partial_{y}, \\
C_{0}^{w, z y}=\partial_{x}, \quad C_{2}^{w, z y}=\left(\partial_{y} f\right)^{2} \partial_{x}-\left(\partial_{x} f\right)\left(\partial_{y} f\right) \partial_{y} .
\end{gathered}
$$

Finally, from (3.13) and (3.14) we find, respectively, the non-zero contributions

$$
\begin{gathered}
\tilde{D}_{1}^{u, x}=\left(\partial_{x} f\right), \quad \tilde{D}_{1}^{u, y}=\left(\partial_{y} f\right), \quad \tilde{D}_{0}^{u, z}=-1, \\
D_{0}^{u, x}=\partial_{x}, \quad D_{2}^{u, x}=\left(\partial_{y} f\right)^{2} \partial_{x}-\left(\partial_{x} f\right)\left(\partial_{y} f\right) \partial_{y}, \\
D_{0}^{u, y}=\partial_{y}, \quad D_{2}^{u, y}=-\left(\partial_{x} f\right)\left(\partial_{y} f\right) \partial_{x}+\left(\partial_{x} f\right)^{2} \partial_{y}, \\
D_{1}^{u, z}=\left(\partial_{x} f\right) \partial_{x}+\left(\partial_{y} f\right) \partial_{y},
\end{gathered}
$$

and

$$
\begin{gathered}
\tilde{D}_{1}^{w, x}=-\left(\partial_{x} f\right), \quad \tilde{D}_{1}^{w, y}=-\left(\partial_{y} f\right), \quad \tilde{D}_{0}^{w, z}=1, \\
D_{0}^{w, x}=\partial_{x}, \quad D_{2}^{w, x}=\left(\partial_{y} f\right)^{2} \partial_{x}-\left(\partial_{x} f\right)\left(\partial_{y} f\right) \partial_{y} \\
D_{0}^{w, y}=\partial_{y}, \quad D_{2}^{w, y}=-\left(\partial_{x} f\right)\left(\partial_{y} f\right) \partial_{x}+\left(\partial_{x} f\right)^{2} \partial_{y}, \\
D_{1}^{w, z}=\left(\partial_{x} f\right) \partial_{x}+\left(\partial_{y} f\right) \partial_{y} .
\end{gathered}
$$

\section{Numerical results}

We now point out that a numerical implementation of this algorithm is immediate. Indeed, we consider a HOPS approach to the solution of (3.16) which amounts to a numerical approximation of solutions to $(4.2)$,

$$
v_{n}=M_{0}^{-1}\left[b_{n}-\sum_{\ell=0}^{n-1} M_{n-\ell} v_{\ell}\right] .
$$

We approximate the members of the truncated Taylor series (4.3),

$$
v_{n}(x, y)=\left(\begin{array}{c}
\tilde{\mathbf{U}}_{n}(x, y) \\
\tilde{\mathbf{W}}_{n}(x, y) \\
\mathbf{U}_{n}(x, y) \\
\mathbf{W}_{n}(x, y)
\end{array}\right)
$$

by

$$
v_{n}^{N_{x}, N_{y}}(x, y):=\sum_{p=-N_{x} / 2}^{N_{x} / 2-1} \sum_{q=-N_{y} / 2}^{N_{y} / 2-1}\left(\begin{array}{c}
\tilde{\mathbf{U}}_{n, p, q} \\
\tilde{\mathbf{W}}_{n, p, q} \\
\mathbf{U}_{n, p, q} \\
\mathbf{W}_{n, p, q}
\end{array}\right) e^{i \alpha_{p} x+i \beta_{q} y},
$$

where $\left\{\tilde{\mathbf{U}}_{n, p, q}, \tilde{\mathbf{W}}_{n, p, q}, \mathbf{U}_{n, p, q}, \mathbf{W}_{n, p, q}\right\}$ are the Fourier coefficients of $\left\{\tilde{\mathbf{U}}_{n}, \tilde{\mathbf{W}}_{n}, \mathbf{U}_{n}, \mathbf{W}_{n}\right\}$, respectively. The unknown coefficients are recovered upon demanding that (4.2) be true for these forms. Convolution products are computed using the FFT algorithm [24] and the only "discretization" is that we restrict to $-N_{x} / 2 \leq p \leq N_{x} / 2-1$ and $-N_{y} / 2 \leq q \leq N_{y} / 2-1$.

Of great importance is how the Taylor series in $\varepsilon,(4.3)$, is to be summed. To be specific, to approximate $v$ we have just considered the truncation

$$
v^{N_{x}, N_{y}, N}(x, y ; \varepsilon):=\sum_{p=-N_{x} / 2}^{N_{x} / 2-1} \sum_{q=-N_{y} / 2}^{N_{y} / 2-1}\left(\sum_{n=0}^{N} \hat{v}_{p, q, n} \varepsilon^{n}\right) e^{i \alpha_{p} x+i \beta_{q} y},
$$


which generates the Taylor polynomials

$$
\hat{v}_{p, q}^{N}(\varepsilon):=\sum_{n=0}^{N} \hat{v}_{p, q, n} \varepsilon^{n}
$$

The classical numerical analytic continuation technique of Padé approximation [4] has been successfully used with HOPS methods on many occasions (see, e.g., $[8,46]$ ), and we recommend its use here. Padé approximation seeks to simulate the truncated Taylor series $\hat{v}_{p, q}^{N}(\varepsilon)$ by the rational function

$$
[L / M](\varepsilon):=\frac{a^{L}(\varepsilon)}{b^{M}(\varepsilon)}=\frac{\sum_{\ell=0}^{L} a_{\ell} \varepsilon^{\ell}}{1+\sum_{m=1}^{M} b_{m} \varepsilon^{m}}
$$

where $L+M=N$ and

$$
[L / M](\varepsilon)=\hat{v}_{p, q}^{N}(\varepsilon)+\mathcal{O}\left(\varepsilon^{L+M+1}\right)
$$

well-known formulas for the coefficients $\left\{a_{\ell}, b_{m}\right\}$ can be found in [4]. This approximant has remarkable properties of enhanced convergence, and we refer the interested reader to $\S 2.2$ of Baker \& Graves-Morris [4] and the insightful calculations of $\S 8.3$ of Bender \& Orszag [5] for a thorough discussion of the capabilities and limitations of Padé approximants.

\subsection{Validation}

For the problem of plane-wave scattering by a (non-flat) diffraction grating there are, of course, no exact solutions. Therefore, in order to validate our code, we compare results of our simulations with those generated from Field Expansions (FE) simulations described and previously verified in the work of the author [44]. Among the myriad choices of quantities to measure we have selected the zeroth ("specular") reflected efficiency, $e_{0,0}^{u}$, as it is the precipitous drop in this quantity which signals the onset of a Surface Plasmon Resonance [52, 34, 19]. This efficiency is computed by an approach outlined in our previous work [43]. The method amounts to introducing additional "artificial boundaries" strictly above and below the uppermost and lowermost interfaces where the Rayleigh expansions (2.4) are valid so that the efficiencies can be trivially recovered.

With this in mind we chose a particular physical configuration which is motivated by the seminal work of Bruno \& Reitich [9] on the FE scheme for simulating the vector Maxwell equations for a doubly layered medium. We consider the sinusoidal biperiodic grating shape specified by

$$
f(x, y)=\frac{1}{4}\left[\cos \left(\frac{2 \pi x}{d}\right)+\cos \left(\frac{2 \pi y}{d}\right)\right],
$$

so that $d_{1}=d_{2}=d$. We chose their wavelength-to-period ratio $\lambda / d=0.83$, but did not restrict to normally incident radiation. Indeed, if we express

$$
\begin{aligned}
\alpha=(2 \pi / \lambda) \nu^{u} \sin (\theta) \cos (\phi), & \beta=(2 \pi / \lambda) \nu^{u} \sin (\theta) \sin (\phi), \\
k^{u}=(2 \pi / \lambda) \nu^{u}, & k^{w}=(2 \pi / \lambda) \nu^{w}, \\
\gamma^{u}=\sqrt{\left(k^{u}\right)^{2}-\alpha^{2}-\beta^{2}}, & \gamma^{w}=\sqrt{\left(k^{w}\right)^{2}-\alpha^{2}-\beta^{2}},
\end{aligned}
$$

where $\nu^{m}$ is the index of refraction of layer $m$, then we chose

$$
\theta=10(2 \pi / 360), \quad \phi=5(2 \pi / 360), \quad \nu^{u}=1, \quad \nu^{w}=2 .
$$

To conclude the specification of the configuration, we selected

$$
\varepsilon / d=0.003,0.01,0.03,0.1, \quad d=0.500 .
$$

For numerical parameters we chose

$$
N_{x}=N_{y}=16, \quad N=10,
$$


and computed the relative error between our new approach (denoted "FIE") and the FE algorithm:

$$
\text { Error }=\frac{\left|e_{0,0}^{u, \mathrm{FIE}}-e_{0,0}^{u, \mathrm{FE}}\right|}{\left|e_{0,0}^{u, \mathrm{FE}}\right|} .
$$

In Figure 2 we study the convergence of our new methodology for various perturbation orders $N$ utilizing Padé approximation as the profile height $\varepsilon$ is varied. In Figure 3 we display results of our

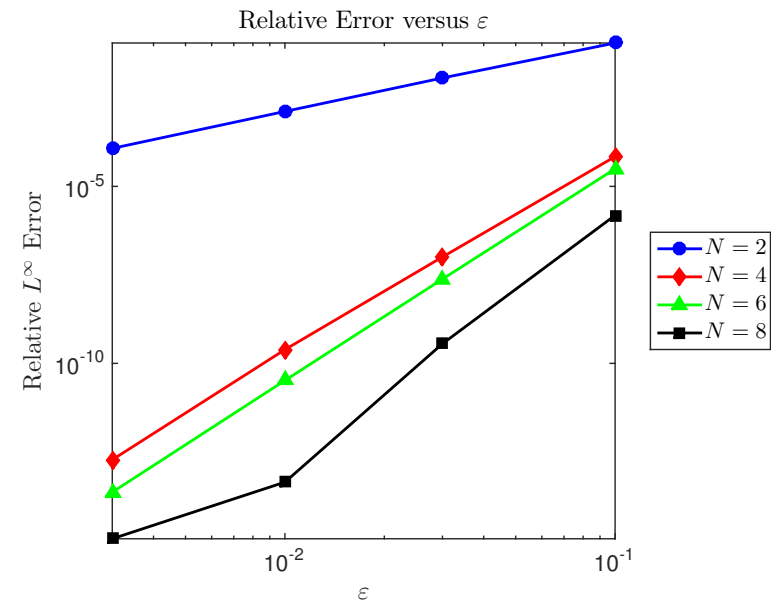

Figure 2: Relative error versus perturbation parameter $\varepsilon$ for various perturbation orders $N$ (with $[N / 2, N / 2]$ Padé approximation). Results for the cosine-cosine configuration, (5.2), with $N_{x}=N_{y}=16$.

convergence study for different values of the height $\varepsilon$ as $N$ is refined. In each of these figures we note

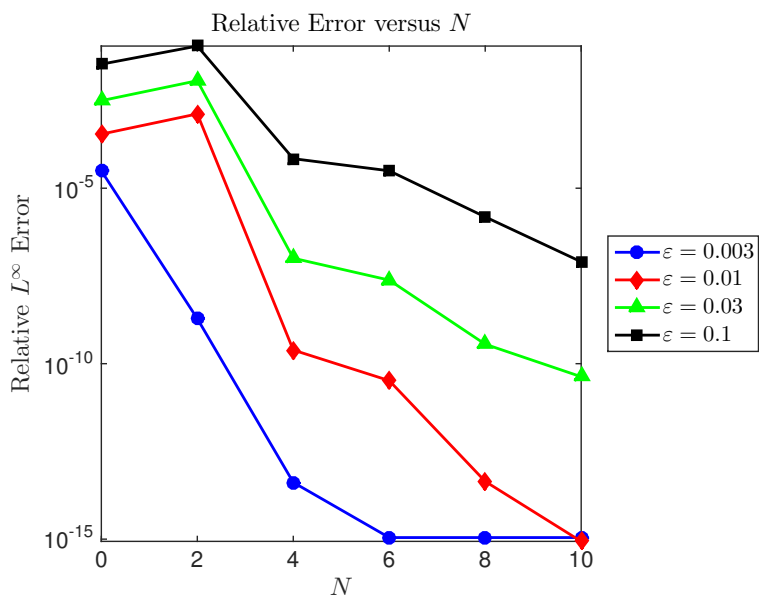

Figure 3: Relative error versus perturbation order $N$ (with $[N / 2, N / 2]$ Padé approximation) for various perturbation parameters $\varepsilon$. Results for the cosine-cosine configuration, (5.2), with $N_{x}=N_{y}=16$.

the rapid and robust convergence of our new approach to the results of the validated, high-order accurate FE methodology [44].

\subsection{Simulation of reflectivity maps}

To conclude, we considered configurations inspired by the simulations of the laboratory of S.H. Oh (Minnesota), particularly the Surface Plasmon Resonance (SPR) sensing devices studied in 
$[33,50]$. In these a two-dimensional sensor was studied featuring a corrugated insulator/conductor interface. While we are unable at present to study the third insulator layer featured there, we are able to add three-dimensional effects and, with very little trouble, change the types of the insulator and/or conductor.

We retained the grating interface shape $f$ defined in (5.2a), chose vacuum as the insulator above the interface so that $\nu^{u}=1$, and filled the lower layer with either gold or silver. The indices of refraction of these metals are the subject of current research, and for these we selected Lorentz models

$$
\epsilon^{\sigma}=\epsilon_{\infty}^{\sigma}+\sum_{j=1}^{6} \frac{\Delta_{j}^{\sigma}}{-a_{j}^{\sigma} \omega^{2}-i b_{j}^{\sigma} \omega+c_{j}^{\sigma}}, \quad \sigma \in\{\mathrm{Au}, \mathrm{Ag}\},
$$

where $\omega=2 \pi / \lambda$, and the parameters $\epsilon_{\infty}^{\sigma}, \Delta_{j}^{\sigma}, a_{j}^{\sigma}, b_{j}^{\sigma}, c_{j}^{\sigma}$ can be found in [53]. For physical and numerical parameters we chose

$$
\begin{gathered}
\theta=0, \quad \phi=0, \\
h=\varepsilon=0, \ldots, 0.200, \quad d_{1}=d_{2}=0.650, \\
N_{x}=N_{y}=12, \quad N=0, \ldots, 8 .
\end{gathered}
$$

In Figure 4 we display the Reflectivity Map, $R(\lambda, h)$, (2.5), for this configuration which shows a strong resonance around $\lambda=670 \mathrm{~nm}$ and $h=90 \mathrm{~nm}$. By contrast, in Figure 5 we display $R,(2.5)$,

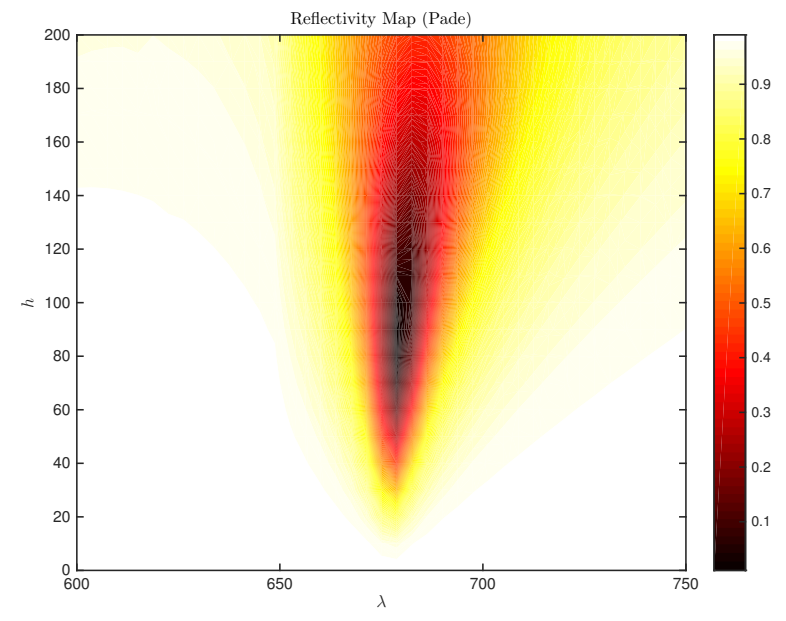

Figure 4: Reflectivity Map for the two-layer vacuum/gold configuration, $R(\lambda, h)$, versus incident wavelength, $\lambda$, and deformation height $h=\varepsilon$. Results for the sinusoidal shape (5.2a) with $N_{x}=N_{y}=12$, [4/4] Padé approximant.

where we have replaced gold with silver. This shows a much more sensitive (narrower in $\lambda$ ) resonance at the new values $\lambda=665 \mathrm{~nm}$ and $h=60 \mathrm{~nm}$.

\section{Acknowledgment}

DPN gratefully acknowledges support from the National Science Foundation through grant No. DMS-1115333

\section{References}

[1] Ablowitz, M. J., Fokas, A. S., Musslimani, Z. H., 2006. On a new non-local formulation of water waves. J. Fluid Mech. 562, 313-343.

[2] Ambrose, D., Nicholls, D. P., 2014. Fokas integral equations for three dimensional layered-media scattering. Journal of Computational Physics 276, 1-25. 


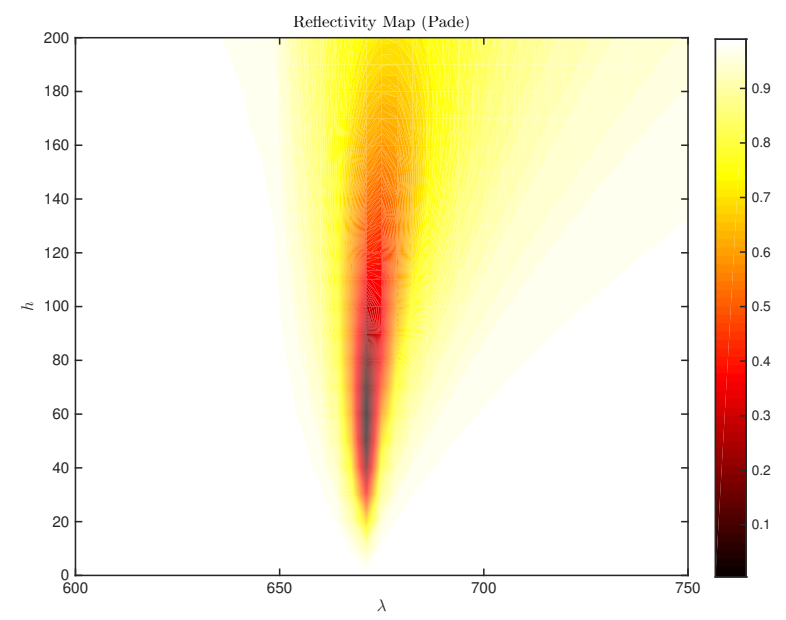

Figure 5: Reflectivity Map for the two-layer vacuum/silver configuration, $R(\lambda, h)$, versus incident wavelength, $\lambda$, and deformation height $h=\varepsilon$. Results for the sinusoidal shape (5.2a) with $N_{x}=N_{y}=12$, [4/4] Padé approximant.

[3] Arens, T., Chandler-Wilde, S., DeSanto, J., 2006. One integral equation and least squares methods for scattering by diffraction gratings. Communications in Computational Physics 6 (1), $1010-1042$.

[4] Baker, Jr., G. A., Graves-Morris, P., 1996. Padé approximants, 2nd Edition. Cambridge University Press, Cambridge.

[5] Bender, C. M., Orszag, S. A., 1978. Advanced mathematical methods for scientists and engineers. McGraw-Hill Book Co., New York, international Series in Pure and Applied Mathematics.

[6] Berenger, J.-P., 1994. A perfectly matched layer for the absorption of electromagnetic waves. J. Comput. Phys. 114 (2), 185-200.

[7] Bruno, O. P., Reitich, F., 1993. Numerical solution of diffraction problems: A method of variation of boundaries. J. Opt. Soc. Am. A 10 (6), 1168-1175.

[8] Bruno, O. P., Reitich, F., 1993. Numerical solution of diffraction problems: A method of variation of boundaries. II. Finitely conducting gratings, Padé approximants, and singularities. J. Opt. Soc. Am. A 10 (11), 2307-2316.

[9] Bruno, O. P., Reitich, F., 1993. Numerical solution of diffraction problems: A method of variation of boundaries. III. Doubly periodic gratings. J. Opt. Soc. Am. A 10 (12), 2551-2562.

[10] Chandler-Wilde, S., Langdon, S., 2015. Acoustic scattering: high frequency boundary element methods and unified transform methods. In: Unified transform method for boundary value problems: applications and advances. SIAM.

[11] Christensen, D., Fowers, D., 1996. Modeling SPR sensors with the finite-difference time-domain method. Biosensors and Bioelectronics 11, 677-684.

[12] Coifman, R., Goldberg, M., Hrycak, T., Israeli, M., Rokhlin, V., 1999. An improved operator expansion algorithm for direct and inverse scattering computations. Waves Random Media 9 (3), $441-457$.

[13] Colton, D., Kress, R., 1998. Inverse acoustic and electromagnetic scattering theory, 2nd Edition. Springer-Verlag, Berlin.

[14] Demesy, G., Zolla, F., Nicolet, A., Commandre, M., 2009. Versatile full-vectorial finite element model for crossed gratings. Optics Letters 34 (14), 2216-2219. 
[15] DeSanto, J., Erdmann, G., Hereman, W., Krause, B., Mirsa, M., Swim, E., 2001. Theoretical and computational aspects of scattering from periodic surfaces: two-dimensional perfectly reflecting surfaces using the spectral-coordinate method. Waves in Random Media 11 (4), 455488.

[16] DeSanto, J., Erdmann, G., Hereman, W., Mirsa, M., 1998. Theoretical and computational aspects of scattering from rough surfaces: one-dimensional perfectly reflecting surfaces. Waves in Random Media 8 (4), 385-414.

[17] DeSanto, J., Erdmann, G., Hereman, W., Mirsa, M., 2001. Theoretical and computational aspects of scattering from periodic surfaces: one-dimensional transmission interface. Waves in Random Media 11 (4), 425-454.

[18] Ebbesen, T. W., Lezec, H. J., Ghaemi, H. F., Thio, T., Wolff, P. A., 1998. Extraordinary optical transmission through sub-wavelength hole arrays. Nature 391 (6668), 667-669.

[19] Enoch, S., Bonod, N., 2012. Plasmonics: From Basics to Advanced Topics. Springer Series in Optical Sciences. Springer, New York.

[20] Fokas, A. S., 2008. A unified approach to boundary value problems. Vol. 78 of CBMS-NSF Regional Conference Series in Applied Mathematics. Society for Industrial and Applied Mathematics (SIAM), Philadelphia, PA.

[21] Givoli, D., 1991. Nonreflecting boundary conditions. J. Comput. Phys. 94 (1), 1-29.

[22] Givoli, D., 1992. Numerical methods for problems in infinite domains. Vol. 33 of Studies in Applied Mechanics. Elsevier Scientific Publishing Co., Amsterdam.

[23] Givoli, D., 1999. Recent advances in the DtN FE method. Arch. Comput. Methods Engrg. 6 (2), $71-116$.

[24] Gottlieb, D., Orszag, S. A., 1977. Numerical analysis of spectral methods: theory and applications. Society for Industrial and Applied Mathematics, Philadelphia, Pa., CBMS-NSF Regional Conference Series in Applied Mathematics, No. 26.

[25] Greengard, L., Rokhlin, V., 1987. A fast algorithm for particle simulations. J. Comput. Phys. $73(2), 325-348$.

[26] Homola, J., 2008. Surface plasmon resonance sensors for detection of chemical and biological species. Chemical Reviews 108 (2), 462-493.

[27] Huber, M., Schoberl, J., Sinwel, A., Zaglmayr, S., 2009. Simulation of diffraction in periodic media with a coupled finite element and plane wave approach. SIAM Journal on Scientific Computation 31 (2), 1500-1517.

[28] Im, H., Lee, S. H., Wittenberg, N. J., Johnson, T. W., Lindquist, N. C., Nagpal, P., Norris, D. J., Oh, S. H., 2011. Template-stripped smooth Ag nanohole arrays with silica shells for surface plasmon resonance biosensing. ACS Nano 5, 6244-6253.

[29] Jackson, J. D., 1975. Classical electrodynamics, 2nd Edition. John Wiley \& Sons Inc., New York.

[30] Jose, J., Jordan, L., Johnson, T. W., Lee, S., Wittenberg, N., Oh, S., 2013. Topographically flat substrates with embedded nanoplasmonic devices for biosensing. Adv Funct Mater 23, 2812 2820 .

[31] Kurkcu, H., Reitich, F., 2009. Stable and efficient evaluation of periodized Green's functions for the Helmholtz equation at high frequencies. J. Comput. Phys. 228 (1), 75-95. 
[32] Lindquist, N., Johnson, T. W., Norris, D., Oh, S.-H., 2011. Monolithic integration of continuously tunable plasmonic nanostructures. Nano Lett. 11, 3526-3530.

[33] Lindquist, N. C., Johnson, T. W., Jose, J., Otto, L. M., Oh, S.-H., 2012. Ultrasmooth metallic films with buried nanostructures for backside reflection-mode plasmonic biosensing. Annalen der Physik 524, 687-696.

[34] Maier, S. A., 2007. Plasmonics: Fundamentals and Applications. Springer, New York.

[35] Malcolm, A., Nicholls, D. P., 2011. A field expansions method for scattering by periodic multilayered media. Journal of the Acoustical Society of America 129 (4), 1783-1793.

[36] Milder, D. M., 1991. An improved formalism for rough-surface scattering of acoustic and electromagnetic waves. In: Proceedings of SPIE - The International Society for Optical Engineering (San Diego, 1991). Vol. 1558. Int. Soc. for Optical Engineering, Bellingham, WA, pp. 213-221.

[37] Milder, D. M., 1991. An improved formalism for wave scattering from rough surfaces. J. Acoust. Soc. Am. 89 (2), 529-541.

[38] Milder, D. M., 1996. An improved formalism for electromagnetic scattering from a perfectly conducting rough surface. Radio Science 31 (6), 1369-1376.

[39] Milder, D. M., 1996. Role of the admittance operator in rough-surface scattering. J. Acoust. Soc. Am. 100 (2), 759-768.

[40] Milder, D. M., Sharp, H. T., 1991. Efficient computation of rough surface scattering. In: Mathematical and numerical aspects of wave propagation phenomena (Strasbourg, 1991). SIAM, Philadelphia, PA, pp. 314-322.

[41] Milder, D. M., Sharp, H. T., 1992. An improved formalism for rough surface scattering. ii: Numerical trials in three dimensions. J. Acoust. Soc. Am. 91 (5), 2620-2626.

[42] Moskovits, M., 1985. Surface-enhanced spectroscopy. Reviews of Modern Physics 57 (3), 783826.

[43] Nicholls, D. P., 2014. A high-order perturbation of surfaces (HOPS) approach to Fokas integral equations: Three-dimensional layered media scattering. Quarterly of Applied Mathematics (to appear).

[44] Nicholls, D. P., 2015. A method of field expansions for vector electromagnetic scattering by layered periodic crossed gratings. Journal of the Optical Society of America, A 32 (5), 701-709.

[45] Nicholls, D. P., Reitich, F., 2001. A new approach to analyticity of Dirichlet-Neumann operators. Proc. Roy. Soc. Edinburgh Sect. A 131 (6), 1411-1433.

[46] Nicholls, D. P., Reitich, F., 2003. Analytic continuation of Dirichlet-Neumann operators. Numer. Math. 94 (1), 107-146.

[47] Nicholls, D. P., Reitich, F., 2004. Shape deformations in rough surface scattering: Cancellations, conditioning, and convergence. J. Opt. Soc. Am. A 21 (4), 590-605.

[48] Nicholls, D. P., Reitich, F., 2004. Shape deformations in rough surface scattering: Improved algorithms. J. Opt. Soc. Am. A 21 (4), 606-621.

[49] Nicholls, D. P., Reitich, F., 2008. Boundary perturbation methods for high-frequency acoustic scattering: Shallow periodic gratings. J. Acoust. Soc. Amer. 123 (5), 2531-2541.

[50] Nicholls, D. P., Reitich, F., Johnson, T. W., Oh, S.-H., 2014. Fast high-order perturbation of surfaces (HOPS) methods for simulation of multi-layer plasmonic devices and metamaterials. Journal of the Optical Society of America, A 31 (8), 1820-1831. 
[51] Petit, R. (Ed.), 1980. Electromagnetic theory of gratings. Springer-Verlag, Berlin.

[52] Raether, H., 1988. Surface plasmons on smooth and rough surfaces and on gratings. Springer, Berlin.

[53] Rakic, A., Djurisic, A., Elazar, J., Majewski, M., 1998. Optical properties of metallic films for vertical-cavity optoelectronic devices. Applied Optics 37 (22), 5271-5283.

[54] Rayleigh, L., 1907. On the dynamical theory of gratings. Proc. Roy. Soc. London A79, 399-416.

[55] Reitich, F., Johnson, T. W., Oh, S.-H., Meyer, G., 2013. A fast and high-order accurate boundary perturbation method for characterization and design in nanoplasmonics. Journal of the Optical Society of America, A 30, 2175-2187.

[56] Reitich, F., Tamma, K., 2004. State-of-the-art, trends, and directions in computational electromagnetics. CMES Comput. Model. Eng. Sci. 5 (4), 287-294.

[57] Rice, S. O., 1951. Reflection of electromagnetic waves from slightly rough surfaces. Comm. Pure Appl. Math. 4, 351-378.

[58] Sai, H., Kanamori, Y., Hane, K., Yugami, H., 2005. Numerical study on spectral properties of Tungsten one-dimensional surface-relief gratings for spectrally selective devices. J. Opt. Soc. Am. A 22, 1805-1813.

[59] Schadle, A., Zschiedrich, L., Burger, S., Klose, R., Schmidt, F., 2007. Domain decomposition method for Maxwell's equations: Scattering off periodic structures. Journal of Computational Physics 226 (1), 477-493.

[60] Spence, E. A., Fokas, A. S., 2010. A new transform method I: domain-dependent fundamental solutions and integral representations. Proc. R. Soc. Lond. Ser. A Math. Phys. Eng. Sci. 466 (2120), 2259-2281.

[61] Spence, E. A., Fokas, A. S., 2010. A new transform method II: the global relation and boundary-value problems in polar coordinates. Proc. R. Soc. Lond. Ser. A Math. Phys. Eng. Sci. 466 (2120), 2283-2307.

[62] Stannigel, K., Konig, M., Niegemann, J., Busch, K., 2009. Discontinuous Galerkin time-domain computations of metallic nanostructures. Optics Express 17, 14934-14947. 